\title{
Creutzfeldt-Jakob disease and infection control
}

\author{
Lynn Johnston MD FRCPC ${ }^{1}$, John Conly MD CCFP FRCPC FACP ${ }^{2}$
}

Ocing ver the past year, several situations have occurred in Canada in which patients who had recently undergone a surgical procedure were subsequently diagnosed with confirmed or suspected Creutzfeldt-Jakob disease (CJD). This raised concerns over contamination of surgical instruments: which instruments might have been contaminated from direct exposure to tissues; can instruments become cross-contaminated by exposure to other contaminated instruments; what assessment is necessary to determine cross-contamination; and what should be done with instruments that have been contaminated. Additionally, should there be a patient traceback in the face of potential but unproven exposure? Unfortunately, there are no easy answers to most of the above questions. Australia, the United Kingdom and the World Health Organization have developed guidelines for the infection control management of patients with CJD, as well as instruments and devices that come into contact with them and their tissues (1-3). Health Canada's draft CJD infection control guidelines, withdrawn from the Health Canada Web site until safety concerns regarding sodium hydroxide can be addressed, closely mirrored recommendations made in those documents. The Centers for Disease Control and Prevention guidelines for CJD are under revision. However, a recent American publication made recommendations on what procedures should be used for reprocessing items that have been in contact with the prion protein (PrP) (4). These recommendations differ substantially from the draft Canadian guidelines. This article reviews current knowledge about CJD, and highlights some of the infection control concerns and controversies.

\section{WHAT IS CJD?}

CJD is one of the transmissible spongiform encephalopathies (TSES), also known as prion diseases. These are fatal degenerative brain diseases that occur in human and certain animal species. The complete structure of the prion has yet to be determined. It was originally thought that the infectious agent was a slow virus or virus-like particle. However, increasing amounts of evidence suggest that unique agents, termed PrPs, are central in the etiology of these diseases (5). The prion is theorized to contain only protein, has no DNA or RNA and replicates by converting the structure of the normal cellular PrP into an abnormal one by a mechanism not yet known (5). The prion diseases are characterized by microscopic intracellular vacuoles (spongiform degeneration), severe astrocytic gliosis and the deposition of prion amyloid plaques in the grey matter of the brain (5). In animals, these diseases include scrapie in sheep and goats, bovine spongiform encephalopathy in cattle, chronic wasting disease in deer and elk, and transmissible mink encephalopathy. In humans, four diseases were originally identified as TSEs: CJD, Gerstmann-Sträussler-Scheinker syndrome, fatal familial insomnia and kuru $(1,5,6)$. Two new forms of human TSEs have been recently identified: variant CJD (VCJD) and sporadic fatal insomnia $(6,7)$. All forms of TSEs are experimentally transmissible, some across species barriers $(5,6,8)$.

Classical CJD is by far the most common human TSE, although still very rare, with an estimated incidence of 0.5 to $1 \mathrm{case} /$ million population/year. The clinical presentation usu-

\footnotetext{
${ }^{1}$ Queen Elizabeth II Health Sciences Centre and Dalhousie University, Halifax, Nova Scotia; ${ }^{2}$ Departments of Pathology and Laboratory Medicine, and Microbial and Infectious Diseases, Centre for Antimicrobial Resistance, Calgary Laboratory Services, Division of Microbiology, University of Calgary, Calgary, Alberta

Correspondence and reprints: Dr Lynn Johnston, Room 5014 ACC, Queen Elizabeth II Health Sciences Centre, 1278 Tower Road, Halifax, Nova Scotia B3H 219. Telephone 902-473-8477, fax 902-473-7394, e-mail ljohnsto@is.dal.ca and Dr John Conly, Departments of Pathology and Laboratory Medicine, and Microbial and Infectious Diseases, Centre for Antimicrobial Resistance, Calgary Laboratory Services, Division of Microbiology, University of Calgary, 1638-10th Avenue SW, Calgary, Alberta T3C 0J5. Telephone 403-209-5338, fax 403-209-5347, e-mail john.conly@cls.ab.ca
} 
ally begins with a progressive dementia that soon becomes associated with cerebellar ataxia, visual deterioration, myoclonus, and a variety of other neurological symptoms and signs. The patient is usually mute and immobile in the terminal stages. In most cases, death occurs within five months of the onset of symptoms of CJD, with $80 \%$ dying within one year (6). It is invariably fatal, and there is no treatment.

Three forms of classical CJD are recognized: sporadic, familial and iatrogenic (infectious). The sporadic form, which compromises $85 \%$ to $90 \%$ of all cases, occurs in the general population with no identifiable cause, and typically affects people between 55 and 75 years of age (3). The median age of death for patients with sporadic CJD is 65 years. The familial form accounts for almost all of the remaining $10 \%$ to $15 \%$. In the familial form, there is generally an earlier age of onset and a more protracted course (6). The iatrogenic form is very rare, accounting for fewer than $1 \%$ of all known cases. It is interesting to note that the clinical presentation of iatrogenic forms may be somewhat different from sporadic CJD. A review of cases of iatrogenic CJD indicated that cerebellar presentations predominated in recipients of pituitary hormone and dura mater grafts (9). Although the clinical presentation can contribute to an increased suspicion that a case is iatrogenic, it cannot definitively distinguish iatrogenic from sporadic CJD. The incubation period for iatrogenic CJD also seems to vary according to how it is acquired. From the observation of cases in which CJD exposure was central (eg, direct application of the CJD agent into the brain during neurosurgery), the incubation periods are relatively short, ranging from 12 to 28 months $(9,10)$. As the inoculation site moves further away from the brain to other tissues, the incubation period is extended. For example, incubation periods ranged from 1.5 to 18 years after exposure to contaminated dura mater (9). Transmission from exposure through a peripheral route (as with human growth hormone [hGH] injections) is associated with an incubation period ranging from five to 30 years (9).

At this time, CJD cannot be readily diagnosed. There is no diagnostic test available using easily accessible biological tissue (11). The diagnosis is currently based on clinical features and supportive investigations, and by postmortem neuropathological examination. A definitive diagnosis is established only by neuropathological examination (12). Characteristic electroencephalogram (EEG) changes such as the presence of triphasic periodic complexes are often of assistance in making the diagnosis (12). However, repeated EEGs may be required before the diagnosis can be made, and the diagnosis cannot be excluded by one normal EEG. Magnetic resonance imaging may show hyperintense signals in the basal ganglia on $\mathrm{T}_{2}$-weighted images (6). A diagnostic test for the detection of $14-3-3$ protein in cerebrospinal fluid (CSF) has high sensitivity (96\%) and specificity (88\%) for sporadic CJD during the clinical illness (11). However, the predictive value for a positive test is much lower. If you consider that there may be a prevalence of $1 \%$, the likelihood of a patient with a positive test having CJD is only $49 \%$. While helpful, the test is most helpful when there is already a strong clinical suspicion that the patient has CJD. The positive predictive value reaches $99 \%$ and the negative predictive value reaches $95 \%$, when the prevalence of disease is $50 \%$ (11). With familial CJD, typical EEG findings are often absent, and the 14-3-3 protein in CSF is found in only about one-half of cases (6).

\section{WHAT IS vCJD?}

vCJD was first recognized in the United Kingdom in 1996, when it was noted by the CJD Surveillance Unit that 10 patients had neuropathological findings clearly distinguishing them from classic CJD (7). As of September 2001, a total of 110 definite and probable cases of vCJD have been identified worldwide: 106 cases in the United Kingdom, three cases in France, and one case in the Republic of Ireland (13-15). So far, no documented cases have been identified in Canada by the Canadian CJD surveillance system (16); also, no cases have been reported in the United States.

vCJD differs in several ways from sporadic CJD. Affected individuals are younger (median age at death 29 years), and the presenting features are often psychiatric and sensory symptoms, followed by other neurological symptoms and progressive cognitive impairment $(8,17)$. It tends to have a relatively longer duration of illness than CJD, ranging from seven to 38 months, with a mean of 14 months (14). The EEGs, while not normal, do not show the 'typical' appearances found in sporadic CJD (7). Magnetic resonance imaging brain scans may show bilateral pulvinar high signals (14). Also, the neuropathology in VCJD differs from sporadic CJD. It is characterized by extensive PrP deposition with florid plaques mainly throughout the cerebrum and cerebellum, surrounded by a zone of spongiform change (7). The abnormal PrP molecular strain type (type 4 ) is unique and distinctive in vCJD patients $(18,19)$. It has commonly been detected in lymphoreticular tissues of these patients, but not in such tissues of patients with other forms of CJD. Hence, vCJD can be diagnosed in the appropriate clinical context by a tonsil biopsy $(18,19)$. PrP was found in the excised appendix of a 48-year-old man who underwent an appendectomy eight months before developing symptoms of vCJD (14). The presence of PrPs in lymphoreticular tissues poses additional infection control issues.

\section{IATROGENIC CJD}

CJD and other human TSEs are not known to spread by contact from person to person, or by the airborne or respiratory route (3). However, transmission can occur during invasive medical interventions (9). Worldwide, as of July 2000, there had been 267 documented transmissions of CJD between humans since the first report in 1974, and new cases continue to appear (9). There are three circumstances in which the transmission of CJD between humans has been demonstrated: transplantation of central nervous system tissue; the use of contaminated instruments during invasive neurological or neurosurgical procedures; and peripheral administration of human pituitary extracts. To date, all known cases of iatrogenic CJD have resulted from exposure to infectious brain, dura mater, or pituitary or eye tissue (4). 
Central nervous system tissue transplants include corneal and dura mater grafts. In 1974, the first case of iatrogenic CJD was reported in the recipient of a corneal graft from a donor who had died of unsuspected CJD (9). Experimental evidence demonstrated that corneas of infected animals could transmit CJD, and that the causative agent spreads along visual pathways (20). To date, three cases of corneal graft-related CJD have been reported, none of them in Canada.

The first case of CJD in a recipient of a cadaveric-derived dura mater transplant was reported in 1987 (21). As of July 2000, 114 cases had been reported worldwide, with four cases occurring in Canada (9). Nearly all contaminated dura mater were produced before 1987 by a single manufacturer whose manufacturing processes were inadequate to inactivate the CJD agent (22). Although the tissue preparation process was modified in 1987, cases from earlier dura mater grafting have continued to appear because of the long incubation period (9). Commercial sources of human dura mater are now closely monitored. However, stringent donor screening and processing of dura mater may not completely eliminate the potential for an infectious graft. Human dura mater is still available in Canada, but hospitals that perform neurosurgery also use synthetic materials, nondural human tissues and animal tissues for dural repair (23).

The first report of CJD transmission to two patients as a result of contact with direct brain instrumentation involving electrodes previously used in the brain of a person with CJD was published in 1977 (24). The electrodes had been reprocessed with $70 \%$ alcohol and formaldehyde vapour $(4,6)$. Support for the conclusion that transmission was iatrogenic followed from studies in which the same recording electrodes were shown to transmit disease to experimental primates (25). Retrospective studies have revealed four other cases of probable iatrogenic transmission that occurred as a consequence of neurosurgical procedures (9). It is presumed that in these cases, the routine sterilization procedures of instruments were insufficient to eliminate infectivity. Some of the transmissions associated with reprocessed instruments occurred several weeks after the instruments were initially exposed to tissues from a CJD patient. No such cases have been reported in Canada. No cases related to neurosurgical instruments have been reported since the 1970s. To date, there has been no evidence of CJD transmission related to neurosurgical instruments used with patients who later developed CJD or who are at risk for CJD but were asymptomatic at the time of surgery (9).

In 1985 , it was recognized that injected human cadaverextracted pituitary growth hormone could transmit CJD to humans (26). Shortly thereafter, it was also recognized that human pituitary gonadotropin administered by injection could transmit CJD between humans (27). As of July 2000, 143 cases of CJD worldwide have been related to growth and gonadotropic hormones of human origin (9). Due to the long incubation period of CJD, it is likely that cases from earlier exposure to hGH will appear in the years to come. In Canada, where hGH was used from 1965 until April 1985, surveillance for hGH-associated CJD has not identified any such cases $(9,28)$.

\section{INFECTION CONTROL ISSUES AND CJD}

Information describing the degree of infectivity for CJD comes from experiments with animal TSEs (6). Different organs and tissues have different infectivity levels in different species $(4,6)$. In general, the higher the concentration of the CJD agent in the tissues of origin, the greater the assumed risk of transmission. In all affected species, infectivity is greatest in brain tissue, is present in some peripheral tissues but generally has been absent from all body fluids except CSF (6). Information regarding human infectivity levels is limited, and is extrapolated from experimental research on animals and rare reports of iatrogenic exposure.

The CJD agent is hardy (as are all TSEs), remains infectious for years in a dried state, and resists all routine sterilization and disinfection procedures commonly used by health care facilities $(3,4,25,29)$. Similar to the situation of assessing infectivity, much of the existing decontamination data has come from studies of TSEs in animal populations (30). The best defined model, scrapie in mice or hamsters, has been repeatedly used in studies to establish practical inactivation procedures. Specific parameters addressed have included the levels of infectivity after exposure to particular chemicals, the temperature range for inactivating the agent by steam sterilization, and comparisons between prevacuum steam (porous load) and gravity displacement sterilizers (30-34). Study results have not been consistent for a variety of reasons: nonstandardized methods with a variety of tissues and varied strains of TSE were chosen for the experiments (eg, intact brain, dried macerated brain tissue or brain homogenates), some strains were more thermostable than others, and varying sample sizes with varying titres of infectivity were used $(4,29,35)$. It has been questioned whether the experimental conditions are relevant to or reflect the clinical situation. Most decontamination studies used high inoculums, tissue homogenates that may protect the prion from inactivation and organic matter on instruments that were not precleaned before the decontamination process (4). It has been suggested that the amounts of tissue used would exceed the bioburden normally found on surgical instruments $(4,31)$. The limitations and conflicting information complicate the extrapolation of these findings to humans and hospital settings.

The current belief is that incineration completely destroys the CJD agent. In the recently withdrawn draft Health Canada guidelines, incineration was recommended as the safest and most unambiguous method for ensuring that there is no risk of residual infectivity on surgical instruments that have become contaminated with the CJD agent. The American recommendations are less conservative in their approach, believing that thorough instrument cleaning removes sufficient proteinaceous material to allow conventional sterilization methods (4). However, evidence suggests that instruments not precleaned before sterilizing may still har- 
bour the CJD agent in the centre of the tissues or fluid remaining on the instruments (9). It is believed that the sterilization process may seal the outer layer of the tissues that remains on the instruments, which consequently protects the CJD agent in the core of these particles (36). Furthermore, studies that evaluate the efficacy of a combination of cleaning and sterilization in the clinical setting have not been published (4).

Chemical processes include the use of either sodium hypochlorite or sodium hydroxide, which have both been found to be effective in decreasing the infectivity of prions $(4,30,34,37)$. In one study, an unexplained and unexpected finding associated with sodium hydroxide was that with bovine spongiform encephalopathy and scrapie agents, $2 \mathrm{~h}$ exposures were less effective than 30 or 60 min procedures (30). In the concentrations required, both of these agents are damaging to instruments and caustic or noxious to health care workers $(38,39)$.

Gravity displacement and prevacuum (porous load) steam sterilizers have been tested using various temperatures and total cycle times $(4,30,34,37)$. However, the results are also contradictory, with some reports showing complete inactivation of the prion and other reports showing incomplete inactivation with both prevacuum (porous load) and gravity displacement steam sterilizers $(4,29,30,35,37)$. While acknowledging the disagreement about the ideal time and temperature cycle, the American publication recommends the following as reasonable on the basis of the scientific literature: $121^{\circ} \mathrm{C}$ to $132^{\circ} \mathrm{C}$ for $60 \mathrm{~min}$ for gravity displacement sterilizers and $134^{\circ} \mathrm{C}$ for $18 \mathrm{~min}$ or longer for prevacuum sterilizers (4). Combination methods are not recommended. The World Health Organization recommends $1 \mathrm{~h}$ for both the gravity and prevacuum sterilizers in combination with a chemical process if the contaminated item is not incinerated (3).

In the face of limited and contradictory findings, and varied recommendations, it may be difficult to know what decision to make when it comes to handling equipment that is (or potentially is) contaminated with PrP. Regardless of what comes in future recommendations, some degree of uncertainty will continue to exist. If cost was not an issue, there would be little difficulty in making a decision. While we await the publication of guidelines, however, preventive and risk management strategies can be considered. Consideration should be given to separately reprocessing instruments that come into contact with high risk tissues from other instruments to minimize the amount of cross-contamination should an initially unrecognized exposure occur. Better methods of instrument tracking are required to facilitate a postexposure workup. A 1998 survey of Canadian hospitals doing neurosurgical procedures found that none of the 19 respondents obtained risk factors for CJD on the preoperative questionnaire, and only 50\% could adequately quarantine instruments if necessary (23). Clinicians and infection control practitioners need to work more closely to identify patients with confirmed or suspect CJD, so that the first clue to the diagnosis does not come when the patient is in the recovery room or three days later when the patient is on the ward (40).

\section{REFERENCES}

1. Creutzfeldt-Jakob Disease and Other Human Transmissible Spongiform Encephalopathies: Guidelines on Patient Management and Infection Control. Canberra: National Health and Medical Research Council, 1996.

2. Advisory Committee on Dangerous Pathogens Spongiform Encephalopathy Advisory Committee. Transmissible spongiform encephalopathy agents: safe working and the prevention of infection. London: The Stationary Office, 1998.

3. World Health Organization. WHO infection control guidelines for transmissible spongiform encephalopathies: report of a WHO consultation. Geneva, March 23 to 26,1999 . < http://www.who.int/emc-documents/tse/ whocdscsraph2003c.html > (Version current at November 16, 2001)

4. Rutala WA, Weber DJ. Creutzfeldt-Jakob disease: recommendations for disinfection and sterilization. Clin Infect Dis 2001;32:1348-56.

5. Prusiner SB. Genetic and infectious prion diseases. Arch Neurol 1993;50:1129-53.

6. Johnson RT, Gibbs CJ. Creutzfeldt-Jakob disease and related transmissible spongiform encephalopathies. N Engl J Med 1998;339:1994-2004.

7. Will RG, Ironside JW, Zeidler M, et al. A new variant of Creutzfeldt-Jakob disease in the UK. Lancet 1996;347:921-5.

8. Collinge J. Variant Creutzfeldt-Jakob disease. Lancet 1999;354:317-23.

9. Brown P, Preece M, Brandel JP, et al. Iatrogenic Creutzfeldt-Jakob disease at the millennium. Neurology 2000;55:1075-81.

10. Brown P, Preece MA, Will RG. "Friendly fire" in medicine: hormones, homografts, and Creutzfeldt-Jakob disease. Lancet 1992;340:24-7.

11. Hsich G, Kenney K, Gibbs CJ, Lee KH, Harrington MG. The 14-3-3 brain protein in cerebrospinal fluid as a marker for transmissible spongiform encephalopathies. N Engl J Med 1996;335:924-30.

12. Human transmissible spongiform encephalopathies. Can Commun Dis Rep 1999;25:1-3,6-7.

13. Department of Health. Monthly Creutzfeldt-Jakob disease statistics. <http://www.doh.gov.uk/cjd/stats/sept01.htm> (Version current at November 10, 2001)

14. Coulthart MB, Cashman NR. Variant Creutzfeldt-Jakob disease: a summary of current scientific knowledge in relation to public health. CMAJ 2001;165:51-8.

15. Birchard K. Variant Creutzfeldt Jakob disease found in Ireland. Lancet 1999;353:2221.

16. Population and Public Health, Health Canada. Surveillance for Creutzfeldt-Jakob disease in Canada. <http://www.hc-sc.gc.ca/hpb/lcdc/bid/bbp/cjd/cjd0999e.html> (Version current at November 10, 2001)

17. Zeidler M, Johnstone EC, Bamber RWK, et al. New variant Creutzfeldt-Jacob disease: psychiatric features. Lancet 1997;350:908-10.

18. Hill AF, Butterworth RJ, Joiner S, et al. Investigation of variant Creutzfeldt-Jakob disease and other human prion diseases with tonsil biopsy samples. Lancet 1999;353:183-9.

19. Hill AF, Zeidler M, Ironside J, Collinge J. Diagnosis of new variant Creutzfeldt-Jacob disease by tonsil biopsy. Lancet 1997;349:99-100.

20. Liberski PP, Yanagihara R, Gibbs CJ, Gajdusek DC. Spread of Creutzfeldt-Jakob disease virus along visual pathways after intraocular inoculation. Arch Virol 1990;111:141-7.

21. Rapidly progressive dementia in a patient who received a cadaveric dura mater graft. MMWR Morb Mortal Wkly Rep 1987;36:49-50,55.

22. Creutzfeldt-Jakob disease associated with cadaveric dura mater grafts - Japan, January 1979-May 1996. MMWR Morb Mortal Wkly Rep 1997;46:1066-9.

23. Leung T, Slinger R, Mindorff C, Ricketts M, Cranston B, Paton S. Creutzfeldt-Jakob disease: infection control policies and neurosurgical instrument processing capabilities in Canadian neurosurgical hospitals. Can J Infect Control 2000:94-7.

24. Bernoulli C, Siegfried J, Baumgartner G, et al. Danger of accidental person-to-person transmission of Creutzfeldt-Jakob disease by surgery. Lancet $1977 ; \mathrm{i}: 478-9$. 
25. Brown P. Transmissible human spongiform encephalopathy (infectious cerebral amyloidosis): Creutzfeldt-Jakob disease, Gerstmann-Sträussler-Scheinker syndrome, and kuru. In: Calne DB, ed. Neurodegenerative Diseases. Philadelphia: WB Saunders, 1994:839-76.

26. Fatal degenerative neurologic disease in patients who received pituitary-derived human growth hormone. MMWR Morb Mortal Wkly Rep 1985;34:359-60,365-6.

27. Cochius JI, Hyman N, Esiri MM. Creutzfeldt-Jakob disease in a recipient of human pituitary-derived gonadotrophin: a second case. J Neurol Neurosurg Psychiatry 1992;55:1094-5.

28. Coulthart MB, Stratton EE, Ricketts MN. Surveillance of Creutzfeldt-Jakob disease in Canada. Can J Infect Dis 1997;8:241-4.

29. Steelman VM. Activity of sterilization processes and disinfectants against prions (Creutzfeldt-Jakob Disease agent). In: Rutala WA, ed. Disinfection, Sterilization and Antisepsis in Health Care. Washington, DC: Association for Professionals in Infection Control and Epidemiology, Inc and Polyscience Publications Inc, 1998:255-71.

30. Taylor DM, Fraser H, McConnell I, et al. Decontamination studies with the agents of bovine spongiform encephalopathy and scrapie. Arch Virol 1994;139:313-26.

31. Taylor DM. Inactivation of SE agents. Br Med Bull 1993;49:810-21.
32. Taylor DM. Decontamination of Creutzfeldt-Jakob disease agent: current strategies. Neuropathol Appl Neurobiol $1991 ; 17: 237$.

33. Taylor DM. Autoclaving standards for Creutzfeldt-Jakob disease agent. Ann Neurol 1987;22:557-8.

34. Ernst DR, Race RE. Comparative analysis of scrapie agent inactivation methods. J Virol Methods 1993;41:193-201.

35. Steelman VM. Creutzfeldt-Jakob disease: decontamination issues. Infect Control Steril Tech 1996:32-9.

36. Taylor DM, Fernie K, Mcconnell I, Ferguson CE, Steele PJ. Solvent extraction as an adjunct to rendering: the effect on BSE and scrapie agents of hot solvents followed by dry heat and steam. Vet Rec 1998;143:6-9.

37. Brown P, Rohwer RG, Gajdusek DC. Newer data on the inactivation of scrapie virus or Creutzfeldt-Jakob disease virus in brain tissue. J Infect Dis 1986;153:1145-8.

38. Mallinckrodt Baker, Inc. Sodium hydroxide material safety data sheet. < http://www.mallchem.com/msds/s4034.htm> (Version current at November 10, 2001)

39. Mallinckrodt Baker, Inc. Sodium hypochlorite solution material safety data sheet. <http://www.jtbaker.com/msds/s4106.htm> (Version current at November 10, 2001)

40. Bauce BC, Berenyi J, Canaon C, et al. Creutzfeldt-Jakob disease guidelines: a regional approach. Can Oper Room Nurs J 2000;18:2-15. 


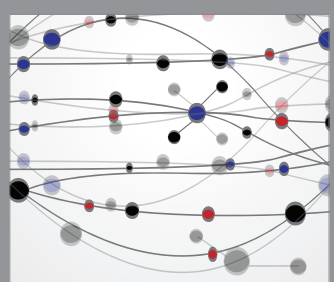

The Scientific World Journal
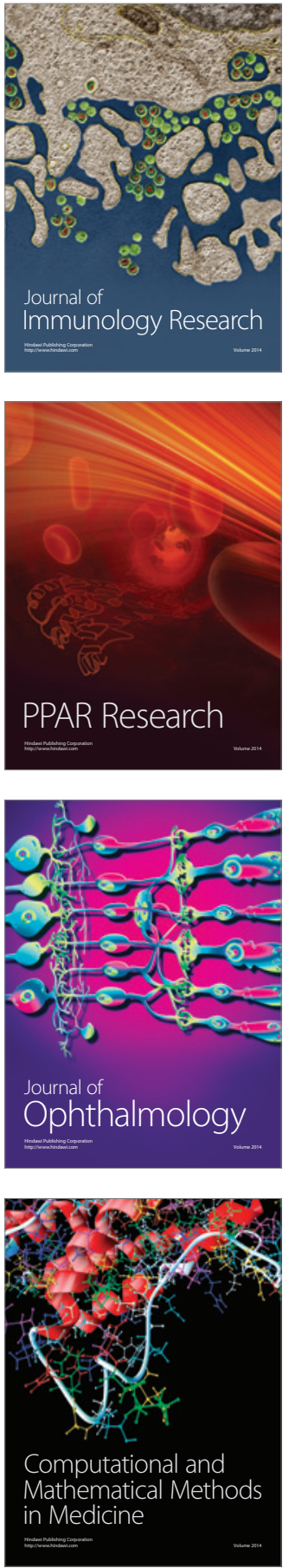

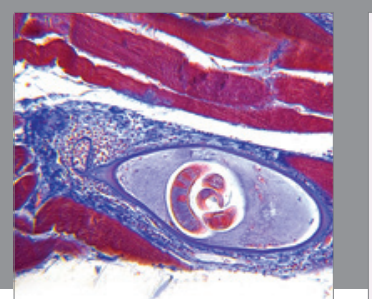

Gastroenterology Research and Practice

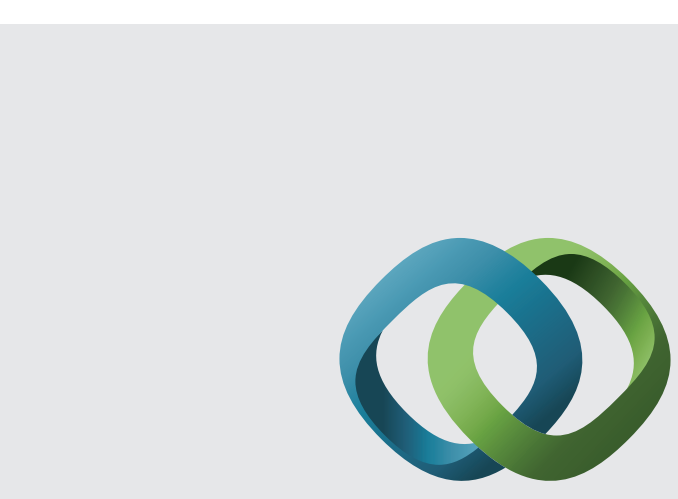

\section{Hindawi}

Submit your manuscripts at

http://www.hindawi.com
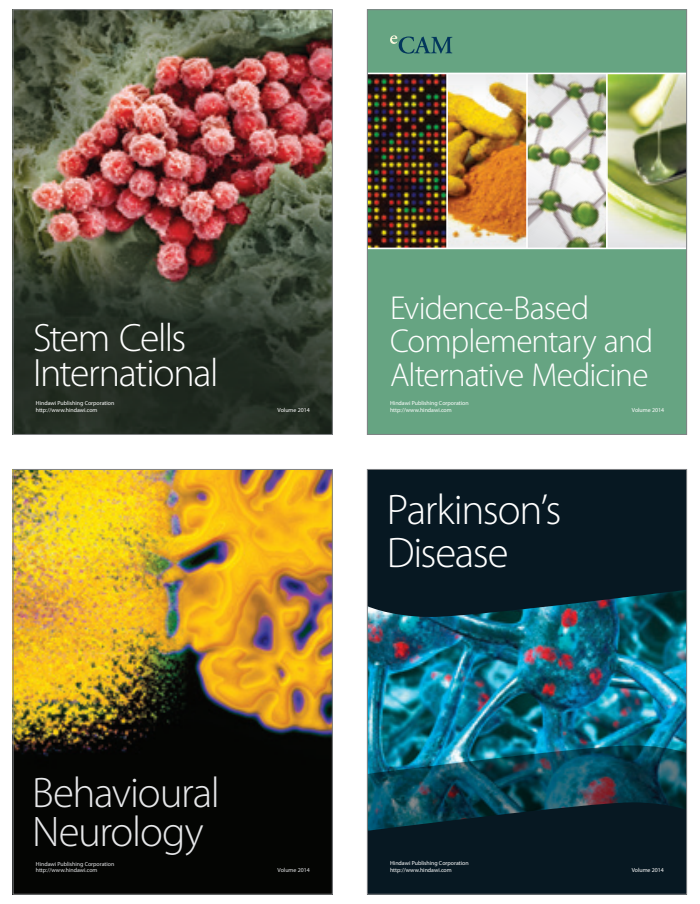
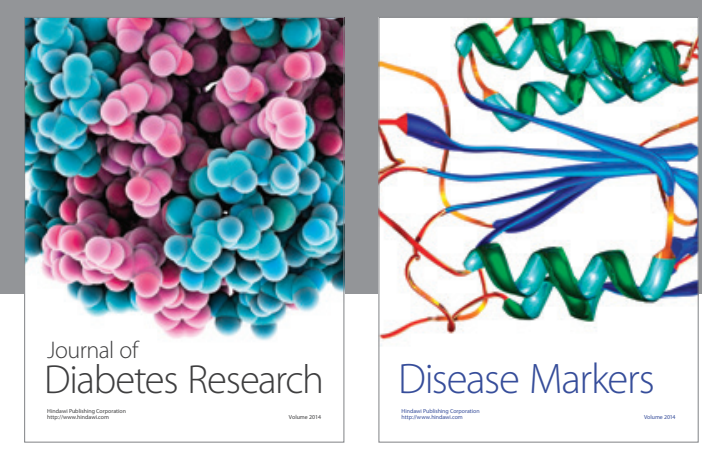

Disease Markers
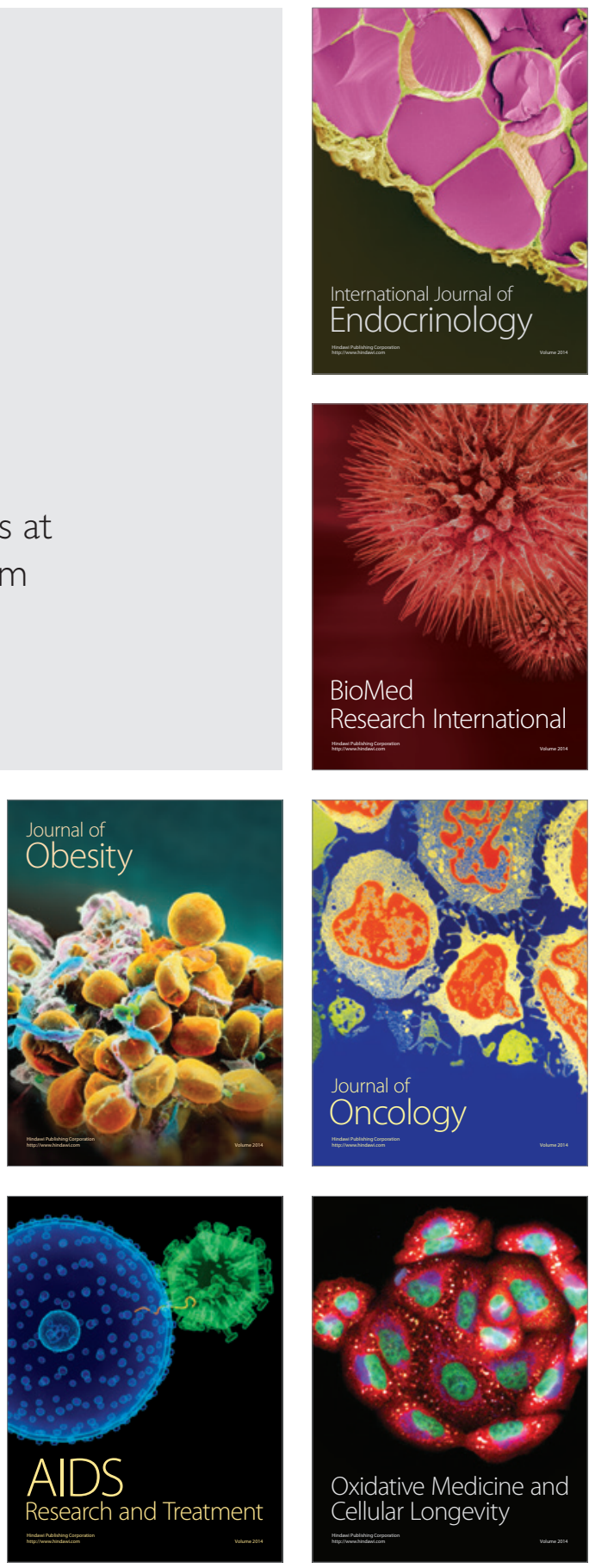